\title{
Electron-Optical Shadow Method of Magnetic-Field Mapping ${ }^{1}$
}

\author{
L. Marton, J. Arol Simpson, and S. H. Lachenbruch
}

\begin{abstract}
The essential features of the electron-optical shadow method for the quantitative mapping of magnetic fields are presented in unified form. The processes of obtaining and analyzing the shadowgrams are given in some detail. Iterative procedures of analysis, which have proved successful in practice, are illustrated by the mapping of an otherwise inaccessible ferromagnetic domain field. Sections 5 and 6 give an analytical method, involving inversion of integral operators. The method, although it has not yet been applied to the analysis of shadowgrams, is given because of its generality and applicability to the equivalent problem of interpretation of fluid dynamic interferograms.
\end{abstract}

\section{Introduction}

A technique was introduced about 3 years ago ${ }^{2,3}$ for quantitative mapping by means of an electronoptical system, of electric- and magnetic-field distributions, which, because of their small dimensions extending to below 1 micron or because they would appreciably be affected by the presence of a probe, are not capable of satisfactory measurement by conventional probe methods. Since that time, both experimental and theoretical studies of this "shadow method" have been made. The purpose of this paper is to summarize briefly in unified form the essential features of the method, as modified and broadened through subsequent published and unpublished studies and experiments.

The shadow method may be considered as consisting of the following three main basic steps: [to be discussed separately]:

Step 1 (Experimental). The field to be studied is placed in the path of an electron beam in the object space of an electron-optical lens system whose

This paper is a summary of 3 years work in the Electron Physics Section of the National Bureau of Standards. Many members, past and present, have contributed to the work described. The bulk of the mathematical development, part of which appears as sections 5 and 6 , is due to S. H. Lachenbruch. In the experimental part, much is due to the work of A. Van Bronkhorst, G. Ford, J. Suddeth, and T. MeCraw.

2 L. Marton and S. H. Lachenbruch, J. Research NBS 43409 (1949) RP2033.

3 L. Marton and S. H. Lachenbruch, J. Appl. Phys. 20, 1171 (1949.)

\section{AXIS OF ROTATION}

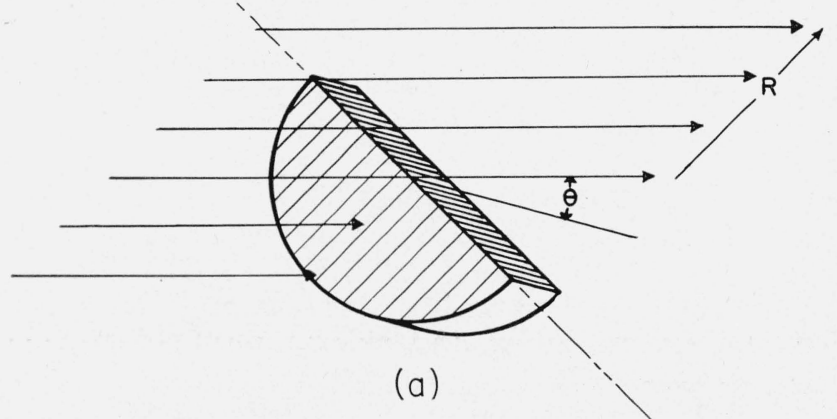

image space contains an opaque object, such as a wire grid, and the distortion of the shadow of this object due to the presence of the unknown field is measured as a function of position. When the field does not have axial symmetry, additional experimental data of value may be obtained by repeating the experiment with different orientations of the field and/or varying beam energies. Figure 1 shows the arrangement of one such nonsymmetrical sample and a representative shadow graph.

Step 2 (Geometrical). From this shadow distortion, and from known geometrical parameters of the electron-optical system, the angular deflections of different rays by the given field are calculated.

Step 3 (Analytical). The field distribution is calculated from this deflection function and from any known or assumed qualitative properties of the unknown field.

\section{Step 1 (Experimental)}

Figure 2 is a schematic diagram of the electronoptical arrangement used. The main components of the system are:

(a) an objective-lens system, characterized by the principal planes, $P, P^{\prime}$, and the focal length, $f$;

(b) the unknown field, placed in object space with its center at a distance $s^{\prime}$, from the first principal

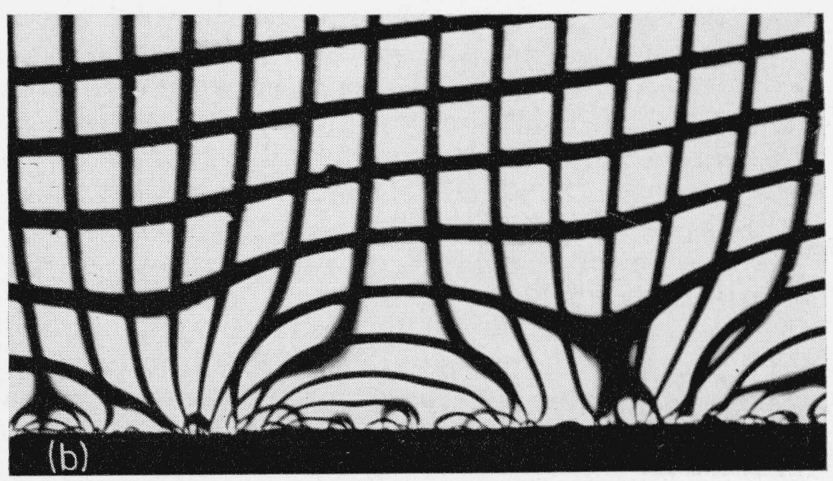

Figure 1.

(a) Typical arrangement for obtaining the shadowgraphs of a nonsymmetrical object. (b) Typical distorted mesh shadows. 


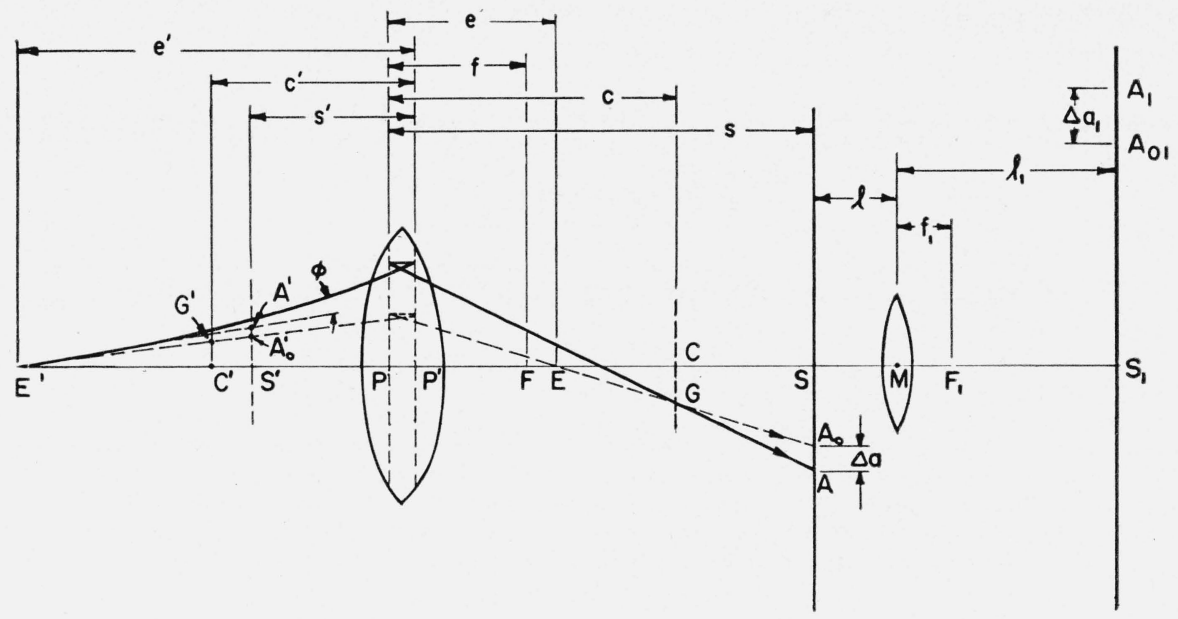

Figure 2. Schematic drawings of the optics of the shadow method.

plane (this determines the conjugate distance $s$ ); (c) a point source, $E^{\prime}$, of electrons, at an "object distance" $e^{\prime}$, (usually $e^{\prime}=\infty$ ), which determines a crossover point, $E$, at a distance $e$ (usually $e=f$ ) in the absence of the unknown field;

(d) a wire mesh or other shadow-casting obstruction, placed in image space at a distance $c$, thereby determining the conjugate distance $c^{\prime}$;

(e) an optional projector lens, $M$, for magnifying the shadow image, characterized by the lens separation, $s+l$, and the focal length, $f_{1}$;

(f) a fluorescent screen or photographic plate in the final image plane $\left(S\right.$ or $\left.S_{1}\right)$ conjugate to the plane containing the center of the field.

Most of the work at the Bureau has been done with a modified ${ }^{4}$ electron microscope, which provides the above components in a convenient form.

As the following conditions are to be assumed in the calculations, it is necessary that they be satisfied experimentally:

Assumption 1. The trajectories within the field lie entirely in one plane (the plane of fig. 2).

Assumption 2. All lateral distances and angles, measured from the optical axis, are sufficiently small to make geometrical aberrations negligible, so the Gaussian dioptrics is applicable.

Assumption 3. The distance between deflecting field and lens field is sufficiently great so that the interaction between the two fields may be neglected.

Assumption 4. The electron energy, $V$, is in the nonrelativistic range.

The assumptions place limitations on the form, position, orientation, and order of magnitude of the electromagnetic field. Thus, assumption 1 requires that planes exist in which the field produces only plane deflections, and assumption 2 requires that these deflections be small. No restriction is placed

4 J. A. Simpson and A. Van Bronkhurst, Rev. Sci. Instr. 21, 669 (1950). on the time variation of the field which may be static or varying. ${ }^{5}$

The solid ray in figure 2 represents a typical electron trajectory, deflected by an angle $\phi$ by the unknown field before entering the lens system, and casting, on the final screen $S_{1}$, a shadow $A_{1}$ of the point $G$ of the opaque obstruction. The broken ray represents the trajectory, which, in the absence of the deflecting field, would pass through this same point $G$, the corresponding shadow being at $A_{01}$. The distance between these two points, which represents the displacement of the shadow of $G$ due to the presence of the field, may be measured for each point $G$, whose shadow can be identified and then be plotted as a function of the distance $a\left(\overline{S_{1} A_{1}}\right)$, which is approximately proportional to the coordinate $a^{\prime}\left(\overline{S^{\prime} A^{\prime}}\right)$ in the deflecting field.

Practically, the distances $\Delta a$ can be measured (1) directly on a plate on which two exposures with and without deflecting field are superimposed; (2) if only one exposure with deflecting field is taken and there exists an undistorted region of the shadow pattern. the straight line corresponding to this part of the plot $\left(a-a_{0}\right)$ versus $a$ can be extrapolated, and the distances between the actually observed portions of the shadow and their extrapolated line can be measured; (3) if no undistorted part of the shadow pattern exists, a second mesh can be introduced between the electron gun (or condensor lens) and the deflecting field providing a reference mesh-wire system. ${ }^{6}$

Tests with the known field in the equatorial plane of a Helmholtz coil as deflecting object have shown that the errors introduced in this $\left(a-a_{0}\right)$ versus $a$ plot by the aberration of the lens and the experimental uncertainties are smaller than 2 percent.

${ }^{5}$ L. Marton and D. L. Reverdin, J. Appl. Phys. 6, 617 (1950)

6 D. L. Reverdin, J. Appl. Phys. 22, 257 (1951). 


\section{Step 2 (Geometrical)}

Assumption 2 above implies the existence of a linear relation between $\Delta a_{1}$, a measurement in the final image plane, and the angle $\phi$, through which the unknown field deflects the ray that passes through the typical point $A^{\prime}$ of the field. This relation may be expressed as

$$
\phi=K \Delta a_{1}
$$

where $K$ is a known function of the magnifications and other fixed geometrical parameters of the system. From the geometry of figure 2 , it is easy to show that $K$ may be expressed as

$$
K=\frac{1}{f \mu_{1}(\nu-1)(1-\epsilon / \mu)},
$$

where

$\mu_{1}=l_{1} / l=\Delta a_{1} / \Delta a=$ magnification of projector system; $\mu=s / s^{\prime}=\Delta a / \Delta a^{\prime}=$ magnification of magnetic object on screen $S$;

$\nu=(s-e) /(c-e)=$ magnification of opaque object $C$ as determined by its shadow on screen $S$ in absence of deflecting field ;

$\epsilon=e / e^{\prime}$ which vanishes when incident beam is parallel.

Whereas in step 1 the deflecting effect of the field was magnified, by means of a lens system and shadow-casting object, in order to facilitate the measurement of that effect, in step 2 this magnified effect, represented by the measurement $\Delta a_{1}$, is converted back to angular deflection in object space.

The problem of experimentally determining $K$ by measuring the quantities on the right-hand side of eq (2) is somewhat complicated by the distortion introduced by the projector lens. This distortion makes the precise determination of the magnifications difficult. A series of tests ${ }^{7}$ on the known field of a small Helmholtz coil shows that with care the errors introduced in the determination of $K$ can be less than 5 percent.

\section{Step 3 (Analytical)}

The discussion of this step will be limited, for simplicity, to magnetic fields, which have been studied in considerable detail.

We note first that assumption 1 above confines the analysis of magnetic fields to planes (such as the equatorial plane) that intersect all magnetic lines of force orthogonally. All electrons initially moving in such a plane will remain therein because their deflection by the field is everywhere normal to both the path and the lines of force. The plane of figure 2 must therefore have this property.

\footnotetext{
7 J. A. Simpson, Proceedings of the NBS Semicentennial Symposium on
} Electron Physies, NBS Circular 527 (1954).
Figure 3, which lies in one such plane, summarizes the theoretical problem of step 3. The polar coordinate system $(r, \theta)$ is attached to the magnetic field $H(r, \theta)$, which is directed normal to the plane of the figure. The relative orientation of field and optical system may be varied experimentally. The curve $L$ represents a typical electron trajectory through the field in this plane. It may be considered as approximately straight because assumption 2 implies that the deflection angle $\phi$ is necessarily small - otherwise the method, which involved geometrical optics, would not have been applicable. Moreover, this assumption eliminates the variation of beam energy as an added parameter for investigation of nonsymmetrical fields.

In steps 1 and 2 a lens system has served the purpose of measuring, by magnifying its effect, the deflection angle, $\phi$, corresponding to each of a certain set of directed lines, $L$, in the plane of figure 3 . Now, each such line is identified uniquely by a pair of coordinates $(p, \beta)$ as shown, where the angle $\beta$ may be varied experimentally by arbitrary plane rotations of the field relative to the lens system, and $p$ is the classical impact parameter, and in the case of parallel illumination is equal to $a_{0} / \mu$. Having served its purposes, therefore, the lens system may now be disregarded, and the problem remaining may be stated as follows: For each of a certain set of directed lines $L=L(p, \beta)$ in the plane of figure 2 , each identified by coordinates $(p, \beta)$, we have an experimentally determined deflection angle $\phi=\phi(p, \beta)$, and hence, by the well-known relation giving the deflection of a charged particle in motion through a magnetic field), the value of

$$
I(p, \beta)=\int_{L(p, \beta)} H(r, \theta) d s=\sqrt{\frac{2 m V}{e}} \phi(p, \beta),
$$

the integral of $H$ along that line. (Here $e, m$, and $V$ are the charge, mass, and potential of the electrons.) We wish to derive from this the magnitude

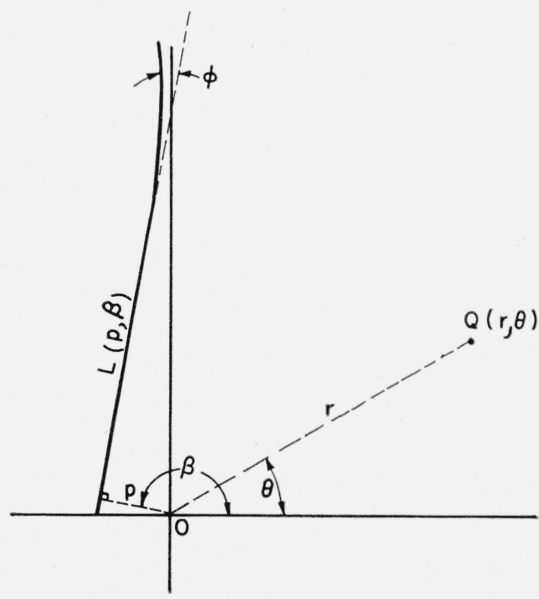

Figure 3. Coordinates used in the analysis of shadowgraphs. 
and sign of the unknown $H$ at an arbitrary point $Q$ with polar coordinates $(r, \theta)$. It is in this stepthat of obtaining the unknown function $H(r, \theta)$ from its integral $I(p, \beta)$ - that a number of procedures have been proposed, and several tried. These methods are summarized below.

It is worth noting that the problem is essentially the same one that arises in the interpretation of interferograms taken of air flow, and any of the methods used in that field apply. Recently, it has been shown that the problem may be programmed for a large-scale computer. ${ }^{8}$ If such a computer is available, this represents the ideal method of analysis of shadowgrams.

For the case where the field is known to have axial symmetry about some axis normal to the plane of figure 3 , we can choose the origin as the intersection of the axis with the plane. Then $H=H(r)$ is independent of $\theta$, and also $I=I(p)$ is independent of $\beta$, so the problem is greatly simplified. Some of the methods about to be described are limited to this case, which, due to the difficulties of the more general case, is the only one that has been investigated in detail up until this time.

\subsection{Method (a). Fitting of parameters}

In the method first used, the unknown field $H(r, \theta)$ is assumed to have a specific analytical form, usually selected from known or predicted physical properties of the field and field-producing object, and involving one or more unknown parameters $\left(a_{1}, a_{2}, \ldots\right)$ to be determined

$$
H(r, \theta)=F\left(r, \theta, a_{1}, a_{2} \ldots a_{n}\right),
$$

where $F$ is a preassigned function of $r$ and $\theta$, periodic in $\theta$.

Integrating the specified function $F$ in advance gives a predicted analytical form for $I$, as a known function $G$ of $(n+2)$ quantities

$I(p, \beta)=\int_{L(p, \beta)} F\left(r, \theta, a_{1}, a_{2} \ldots a_{n}\right) d s=G\left(p, \beta, a_{1} \ldots a_{n}\right)$

We wish to solve for the $n$ quantities $a_{i}$ by matching this $G$-function against the values of the $I$-function obtained experimentally for different values of $p$ and $\beta$.

One way to do this is statistical - by applying least squares to the experimental results, starting with an equation of the form

$$
\sum_{k=1}^{m}\left[I_{k}-G\left(p_{k}, \beta_{k}, a_{1} \ldots a_{n}\right)\right]^{2}=\text { minimum, }
$$

$m$ being the number of electron paths for which

${ }^{8}$ F. D. Bennett et al. Interferometric analysis of airflow about projectiles in free flight, Ballistic Research Laboratories, Aberdeen Proving Ground, Report No. 797 (March 1952). experimental data have been obtained. Here the closeness of fit can usually be used as a partial check on the validity of the functional form $F$ chosen. But how good a check is not known until we consider the question of uniqueness, mentioned at the conclusion of section 6 .

An example of the method of fitting of parameters is described in detail in an earlier paper (see footnote 3). The field of a magnetized wire, in its equatorial plane, was evaluated on the hypothesis that its form approximated that of the field between two equal and opposite point poles of unknown magnetic moment, $a^{1}$, separated by an unknown distance, $2 a_{2}$. For this case, the function $F$ of eq (4) is

$$
H(r)=F\left(r, a_{1}, a_{2}\right)=a_{1}\left(r^{2}+a_{2}^{2}\right)^{-3 / 2} .
$$

The corresponding function $G$ is found from eq (5) to be

$$
G\left(p, a_{1}, a_{2}\right)=2 a_{1} /\left(p^{2}+a_{2}^{2}\right) .
$$

The moment $a_{1}$ and pole separation, $2 a_{2}$, and hence the field $H(r)$, were obtained by a least-squares method of fitting the function $G$ to an experimental scatter diagram derived from deflection measurements.

\subsection{Method (b). Evaluation by step-function approximation}

In a second method, the field is assumed to exist only in a finite portion of the plane of figure 3 , and this portion subdivided into $n$ regions $R_{k}$ of chosen shape and size, and the field calculated as if it were a step-function constant within each such region

$$
H(r, \theta) \equiv a_{k}
$$

where $(r, \theta) \epsilon R_{k}, k=1,2 \ldots n$. By integration we obtain

$$
I(p, \beta)=\sum_{k=1}^{n} a_{k} \lambda_{k}(p, \beta),
$$

where $\lambda_{k}(p, \beta)$ is defined to be the length of the segment of intersection of the line $L(p, \beta)$ and the region $R_{k}$.

If, then, $I(p, \beta)$ is known experimentally for $n$ different electron paths $L(p, \beta)$, there result $n$ simultaneous linear equations in the $n$ constant values $a_{k}$ of $H$. These equations may, in general, be solved for the $a_{k}$,although the labor may be great in many cases. Moreover, it is not possible generally to choose a grid and a set of rays that insure that the set of equations is linearly independent. This proves to be a very serious difficulty.

The technique to be described avoids both difficulties. Figure 4 shows the grid used to study the field about a corner of the cobalt crystal of figure 1 . This is merely an illustration, and a number of other grids have been developed for specific problems, but all use the same technique. The rays are so 

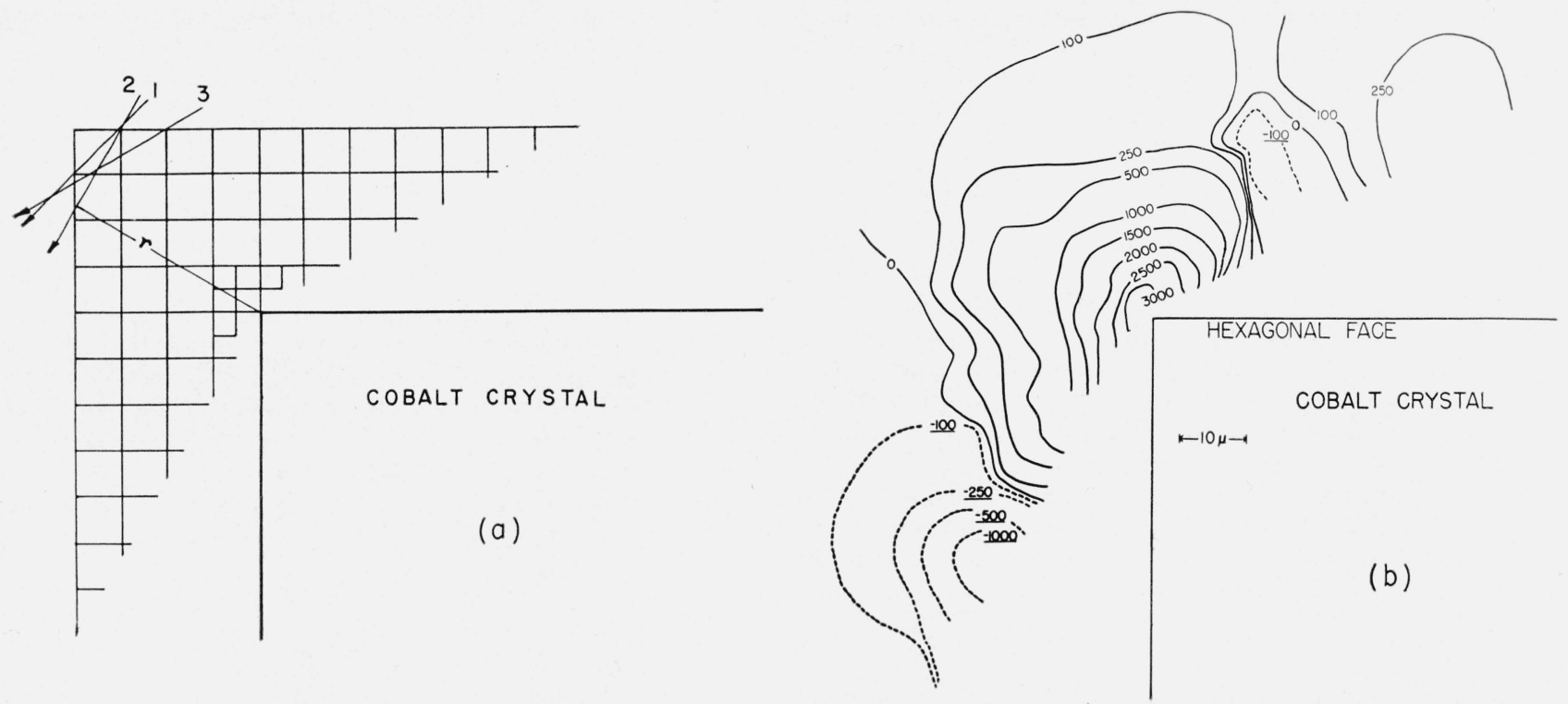

FIgURE 4. Analysis of magnetic fringe field of a cobalt crystal.

(a) Grid used in evaluation of fields about the corner of the sample by means of the step-function approximation. (b) Results of this method on the fringe field of a single cobalt crystal.

chosen that each ray passes through only one square for which $a_{k}$ has not previously been determined. The first three such rays are shown in figure 4 . With such a system, the determinant is triangular and can be solved within a reasonable time. Unfortunately, with a given number of values of the angular parameter, only a limited number of grids may be used before it is impossible to draw a line that does not pass through two or more unknown grids. In the grid illustrated, this takes place after 47 squares have been evaluated, when there are shadowgraphs available for each $15^{\circ}$ of revolution. Figure $4, \mathrm{~b}$, shows the results of this method applied to the field of the cobalt crystal

The errors inherent in this procedure are complicated and large. In addition to any experimental errors involved, one has the error caused by replacing the integral with the rather crude sum. This error can be estimated by changing the number of the meshes up to the maximum possible and observing the change in values. A value for this error seems, in practice, to be as large as several hundred percent. The exact value depends on the magnitude of the gradient and also on the sensitivity of the original shadowgraph. (The field is considered to fall to zero outside the grid, which, in practice, is extended to the limit of the region in which there is a measurable deflection.)

Known properties of the field sometimes suggest a special choice of subdivision. For example, if the field is known to have rotational symmetry about some known point in the plane of figure 3, the most appropriate mode of subdivision would be into rings bounded by concentric circles about that point, inasmuch as the field is constant along any one of these circles.
The field surrounding a magnetized wire has been computed by this method and showed good agreement with the result obtained by method (a), in which the field was assumed to be that of a pair of point poles (see eq 8 and 9 ).

\subsection{Method (c). Series expansion}

A field that is known to have axial symmetry about a point at which it may reasonably be assumed to become infinite can, in general, be assumed expansible as a series in inverse powers of the distance $r$ from that point, such a series being convergent for sufficiently large $r$

$$
H(r)=\frac{a_{2}}{r^{2}}+\frac{a_{3}}{r^{3}}+\cdots
$$

Physically, this expansion corresponds to assuming that the field is that of an $n$-pole, and hence no terms below that corresponding to a single pole $\left(a_{z}\right)$ appears.

$\mathrm{By}$ integration of (12) along the horizontal line $L(p, \pi / 2)$ whose equation is $r \sin \theta=p$, we obtain

$$
\begin{aligned}
I(p) & =\int_{L(p, \pi / 2)} H(r) d s=\int_{L} H(r) d\left(\sqrt{r^{2}-p^{2}}\right) \\
& =2 \int_{p}^{\infty}\left(\frac{a_{2}}{r}+\frac{a_{3}}{r^{2}}+\cdots\right) \frac{d r}{\sqrt{r^{2}-p^{2}}} \\
& =\frac{\pi a_{2}}{p}+\frac{2 a_{3}}{p^{2}}+\cdots
\end{aligned}
$$

On matching this series expression for $I(p)$ against the function obtained experimentally, one is able to 
evaluate numerically the coefficients $a_{2}, a_{3}, \ldots$.., and hence deduce the series representation (12) for $H$.

Even when the condition of axial symmetry is not satisfied, it may sometimes be possible to find types of series appropriate for expansion of given field distributions. The numerical evaluation of the coefficients would then be obtained, as above, by matching the termwise integral of the series with the experimentally derived function $I(p, \beta)$. The restrictions, techniques, etc., would of course vary from case to case; thus, for example, if a Fourier series, or other series in orthogonal functions, is used, the unknown coefficients will generally be obtainable from an explicit formula involving definite integrals.

The series expansion method, although it has a certain amount of generality, must be used with some caution. On account of questions of convergence, for example, a series of form (12) could never be used in the neighborhood of the point $r=0$.

\section{Direct Method of Inversion of Integral Operators}

It is to be noted that in all three methods described above, the approach consists in assuming a priori some specific analytical form for $\mathrm{H}$, involving one or more unknown parameters, which are then evaluated from experimental results. In this sense, they may all, in fact, be regarded as special cases of method (a). If, for example, the function $F$ of (4) is taken to be the step function of (10), the result is essentially method (b); and if $F$ is a series such as (12), one obtains method (c). Still other methods may be derived by choosing $F$ as a function of still different form. Thus a generalization of method (b) is obtained by choosing $F$ to be a linear or seconddegree function, rather than a constant, in each region $R_{k}$.

An entirely different approach consists in interpreting (3) as an integral equation, and observing that it can, in general, be uniquely solved for the unknown function $H(r, \theta)$ in terms of the function $I(\mathrm{p}, \beta)$ which may be obtained experimentally.

To date, no use has been made of the method in experimental work. However, because of its generality, preciseness, and application to the analysis of interferograms in fluid dynamics, it is given in detail.

\subsection{Analytical solution for case of axial symmetry}

For the following considerations, the integral equation (3) may be expressed in a more specific form by using the equation of $L(p, I)$ (see fig. 3 ).

$$
P=r \cos (\theta-\beta) .
$$

By solving (14) for $\theta$, we see that, on the line $L(p, \beta)$

$$
\theta= \begin{cases}\beta+\cos ^{-1} p / r & \text { on the one half-line } \\ \beta-\cos ^{-1} p / r & \text { on the other half line }\end{cases}
$$

Substituting (15) into (3), and expressing $d s$ as a function of $r$, we obtain

$$
\begin{aligned}
I(p, \beta)= & \int_{p}^{\infty}\left[H\left(r, \beta+\cos ^{-1} \frac{p}{r}\right)+\right. \\
& \left.H\left(r, \beta-\cos ^{-1} \frac{p}{r}\right)\right] \frac{r d r}{\sqrt{r^{2}-p^{2}}} .
\end{aligned}
$$

We take first the important special case where the field is known to have axial symmetry about some axis normal to the plane $P$. We can choose as origin of coordinates the intersection of this axis with $P$. Then $H=H(r)$ is independent of $\theta$, and also $I=I(p)$ is independent of $\beta$. Hence, (16) simplifies to the form

$$
I(p)=2 \int_{p}^{\infty} H(r) r d r / \sqrt{r^{2}-p^{2}} .
$$

Now Abel's integral equation,

$$
f(z)=\int_{a}^{z} g(y) d y /(z-y)^{\lambda} \quad(0<\lambda<1)
$$

is known to have the unique continuous solution

$$
g(y)=\frac{\sin \lambda \pi}{\pi} \frac{d}{d y} \int_{a}^{y} f(z) d z /(y-z)^{1-\lambda},
$$

provided $f(z)$ satisfies certain very general continuity conditions and $f(a)=0$. If in $(18)$ one replaces $z$ by $\left(-p^{2}\right), f(z)$ by $I(p), a$ by $-\infty, \lambda$ by $\frac{1}{2}$ by $2, y$ by $\left(-r^{2}\right)$, and $g(y)$ by $H(r)$, it is seen to reduce exactly to (17). By (19), therefore, the unique solution of integral eq (17) is

$$
H(r)=\frac{\sin \pi / 2}{\pi} \frac{d}{d\left(-r^{2}\right)} \int_{-\infty}^{-r^{2}} I(p) d\left(-p^{2}\right) / \sqrt{p^{2}-r^{2}},
$$

which may be reduced to any of the following equivalent forms

$$
\begin{aligned}
H(r) & =\frac{-1}{\pi r} \frac{d}{d r} \int_{r}^{\infty} I(p) p d p / \sqrt{p^{2}-r^{2}} \\
& =\frac{-1}{\pi r} \int_{0}^{\infty} \frac{\partial}{\partial r}\left[I\left(\sqrt{r^{2}+\mu^{2}}\right)\right] d \mu \\
& =-\frac{1}{\pi} \int_{0}^{\infty} I^{\prime}\left(\sqrt{r^{2}+\mu^{2}}\right) d \mu / \sqrt{r^{2}+\mu^{2}} \\
& =-\frac{1}{\pi} \int_{r}^{\infty} I^{\prime}(p) d p / \sqrt{p^{2}-r^{2}} .
\end{aligned}
$$

We have thus an explicit expression for the field $H(r)$ in terms of the empirically known function $I(p)$. If $I(p)$ is known at a sufficient number of experimental points so that a curve may be plotted, the value of $H(r)$ corresponding to each value of $r$ may be calculated directly from (20) by some numerical integration procedure. 
The integral eq (17) and its solution (20) may be abbreviated as

$$
I(p)=T\{H(r)\} ; \quad H(r)=T^{-1}\{I(p)\} .
$$

This form suggests a somewhat different way of viewing the problem: viz, as the problem of inverting a linear integral operator $T$ in function-space.

It can also be considered as a problem in integral transforms. When miscellaneous elementary functions are substituted for $H(r)$, and the corresponding functions $I(p)$ are calculated from (17) and simplified, and then verified by substitution into (20), a table of transforms may be constructed, similar in form to a table of Fourier or Laplace transforms, and the linearity property may be used to derive the transforms of more complicated functions, including the field-distribution functions most frequently encountered.

The one-to-one correspondence implied by (21), or by (17) and (20), provides a conclusive answer, for the axially symmetric case, to the question of uniqueness of the result - a question not rigorously investigated previously. We can now state that if the shadow method is applied to a magnetic field known to have axial symmetry, a ad if a sufficient number of experimental measurements are made so that the empirical function $I(p)$ may be considered as a known continuous function of the continuous variable $p$, then there exists one and only one (continuous) function $H(r)$ satisfying (17), and it is given in terms of the known $I(p)$ by $(20)$.

\subsection{Analytical solution for fields of general form}

Finally, we turn to the general case, removing the condition of axial symmetry. The general problem is that of solving (16) for the function $H(r, \theta)$ of two variables, when sufficient experimental data are available so that $I(p, \beta)$ may be treated as an experimentally known function of both variables.

We note first that for any fixed value of $r$, the field $H$ must be a periodic function of $\theta$, with period $2 \pi$, and so it must have a unique Fourier series expansion with functions of $r$ as coefficients

$$
H(r, \theta)=\sum_{n=-\infty}^{+\infty} H_{n}(r) \exp (i n \theta)
$$

Similarly, $I$ has a unique Fourier expansion, with functions of $p$ as coefficients

$$
I(p, \beta)=\sum_{-\infty}^{+\infty} I_{n}(p) \exp (i n \beta) .
$$

On substituting (22) and (23) into (16), and equating coefficients of like powers of $\exp (i \beta)$ on the two sides of the resulting equation, one obtains

$$
\begin{gathered}
I_{n}(p)=2 \int_{p}^{\infty} H_{n}(r) \cos \left(n \cos ^{-1} p / r\right) \frac{r d r}{\sqrt{r^{2}-p^{2}}} \\
(n=0, \pm 1, \pm 2, \ldots) .
\end{gathered}
$$

For each value of $n,(24)$ is an integral equation for $H_{n}(r)$ in terms of $I_{n}(p)$.

A calculation carried out in the appendix shows that these integral equations have unique solutions for the $H$-coefficients in terms of the corresponding $I$-coefficients. If, then, one has sufficient experimental data to define the continuous function $I(p, \beta)$, the coefficients in its Fourier expansion (23) are uniquely determined from the formula

$$
I_{n}(p)=\frac{1}{2 \pi} \int_{0}^{2 \pi} I(p, \beta) \exp (-i n \beta) d \beta,
$$

the coefficients $H_{n}(r)$ are then determined in turn as solutions of the integral eq (24), and so the desired function $H(r, \theta)$ will be obtained explicitly in the form of a Fourier series (22).

The fact that a Fourier series representation was used here as a tool to solve the general problem, and to investigate existence and uniqueness questions, may tend to obscure the basic nature of the result. By analogy with (21), we may abbreviate the integral eq (16) and its solution as

$I(p, \beta)=T(H(r, \theta)), \quad$ and $\quad H(r, \theta)=T^{-1}(I(p, \beta))$.

This form again suggests that the problem may be viewed as that of inverting a linear integral operator $T$, this time in a space of functions of two variables. It is possible that more compact expressions and proofs might be possible when the problem is attacked from such a point of view.

The question of uniqueness is again seen to be conclusively answered as a by-product of this analysis - this time for the general (nonsymmetric) case. We can state that if, in applying the shadow method to a magnetic field that is not known to have axial symmetry, sufficient experimental data are obtained so that the function $I(p, \beta)$ may be considered as a known continuous function of the two continuous variables $p$ and $\beta$, periodic in $\beta$ (with period $2 \pi$ ), then there exists one and only one (continuous) function $H(r, \theta)$, periodic in $\theta$ (with period $2 \pi$ ), and satisfying (3). It is obvious that in order to satisfy the requirement of sufficient data in this general case, it will be necessary to determine the dependence of $I$ on $\beta$ by repeating the experimental measurements for different relative orientations of field and optical axis.

\section{Appendix. Outline of a Procedure for the successive solution of the integral equations (24)}

(a) When $n=0$, (24) becomes

$$
I_{0}(p)=2 \int_{p}^{\infty} H_{0}(r) d r / \sqrt{r^{2}-p^{2}} .
$$

which has exactly the same form as (17), and, therefore, by (20), has the unique continuous solution

$$
H_{0}(r)=-\frac{1}{\pi} \int_{r}^{\infty} I_{0}^{\prime}(p) / \sqrt{p^{2}-r^{2}} .
$$


(b) When $n= \pm 1,(24)$ takes the form

or

$$
I_{ \pm 1}(p)=2 p \int_{p}^{\infty} H_{ \pm 1}(r) d r / \sqrt{r^{2}-p^{2}}
$$

$$
I_{ \pm 1}(p) / p=2 \int_{p}^{\infty} \frac{H_{ \pm 1}(r)}{r} \frac{r d r}{\sqrt{r^{2}-p^{2}}},
$$

which is of the same form as $(27)$ except that the functions $I_{0}(p)$ and $H_{0}(r)$ are replaced, respectively, by $I_{ \pm:}(p) / p$ and $H_{ \pm 1}(r) / r$. The unique solution, obtained from $(20)$, is therefore

$$
H_{ \pm 1}(r)=-\frac{r}{\pi} \int_{r}^{\infty} \frac{d}{d p}\left[\frac{I_{ \pm 1}(p)}{p}\right] \frac{d p}{\sqrt{p^{2}-r^{2}}}
$$

(c) The higher-order coefficients (for $|n| \geq 2$ ) are more difficult to evaluate. The following method of obtaining the coefficients for $n= \pm 2$ and $n= \pm 3$ appears capable of extension to coefficients of higher order.

Since

$\cos \left[ \pm 2 \cos ^{-1} k\right]$

$$
\equiv 2\left(k^{2}-1\right)+1 ; \quad \cos \left[ \pm 3 \cos ^{-1} k\right] \equiv 4 k\left(k^{2}-1\right)+k
$$

(24) becomes, for $n= \pm 2$ or \pm 3 ,

$I_{n}(p)=2 \int_{p}^{\infty} H_{n}(r)\left(\frac{p}{r}\right)^{|n|-2}\left\{A_{n}\left[\left(\frac{p}{r}\right)^{2}-1\right]+1\right\} \frac{r d r}{\sqrt{r^{2}-p^{2}}}$,

where

$$
A_{2}=A_{-2}=2, A_{3}=A_{-3}=4
$$

But (32) may be written as

$$
\frac{I_{n}(p)}{p^{|n|-2}}=2 \int_{p}^{\infty} \frac{H_{n}(r) r d r}{r^{|n|-2} \sqrt{r^{2}-p^{2}}}-2 A_{n} \int_{p}^{\infty} \frac{H n^{(r)}}{r^{|n|-1}} \sqrt{r^{2}-p^{2}} d r,
$$

which becomes, on applying integration by parts to the last integral,

where

$$
\frac{I_{n}(p)}{p^{|n|-2}}=2 \int_{p}^{\infty} f_{n}(r) \frac{r d r}{\sqrt{r^{2}-p^{2}}}
$$

$$
f_{n}(r)=\frac{H_{n}(r)}{r^{|n|-2}}-A_{n} \int_{r}^{\infty} \frac{H_{n}(\mu) d \mu}{\mu^{|n|-1}} .
$$

Equation (35) is again of the general form (17) with certain replacemen s, and hence, by $(20)$

$$
f_{n}^{*}(r)=-\frac{1}{\pi} \int_{r}^{\infty} \frac{d}{d p}\left[\frac{I_{n}(p)}{p^{|n|-2}}\right] \frac{d p}{\sqrt{p^{2}-r^{2}}} .
$$

Substituting (36) into (37) and differentiating with respect to $r$,

$\frac{d}{d r}\left[\frac{H_{n}(r)}{r^{|n|-2}}\right]+\frac{A_{n}}{r}\left[\frac{H_{n}(r)}{r^{|n|-2}}\right]=\frac{-1}{\pi} \frac{d}{d r} \int^{\infty} \frac{d}{d p}\left[\frac{I_{n}(p)}{p^{|n|-2}}\right] \frac{d p}{\sqrt{p^{2}-r^{2}}}$

But (38) is a linear first-order differential equation in the unknown function $H_{n}(r) / r^{|n|-2}$, which can be solved explicitly for $H_{n}(r)$ by the usual formula, and the arbitrary constant evaluated from the condition that it vanishes at $r=\infty$. This determines the functions $\mathrm{H}_{2}, \mathrm{H}_{-2}, \mathrm{H}_{3}, \mathrm{H}_{-3}$ uniquely, as was to be proved.

\section{Summary}

The electron-optical shadow method of field mapping has been used at the Bureau for 3 years to study such diverse and otherwise inaccessible fields as the fringe fields of ferromagnetic domains of single crystals and collodial particles, space-charge fields in magnetrons, and the external fields of magnetic recording wire. The over-all accuracy of the method depends upon the analysis of the experimental data, and, in favorable cases, has been as good as 5 percent although 10 or 100 times greater error may result in the more unfavorable cases. But in any case, the method opens a new magnitude of fields to study and measure.

In addition to those mentioned in the text and notes, the following nembers, past and present, in the Electron Physics Section, National Bureau of Standards, have by their efforts advanced the work reported: H. Mendlowitz, H. Ernst, J. Stewart, A. A. Nargizian, D. Schubert, P. Weinzierl, L. Greene, A Dauses, and L. Mann. We express our appreciation for their help.

Washington, March 17, 1953. 